\title{
Signs of tracheobronchitis may constitute the principal finding on the lung SPECT/CT images of COVID-19 patients
}

\author{
Achraf Bahloul $^{1} \cdot$ Antoine Verger ${ }^{1,2} \cdot$ Damien Mandry $^{2,3} \cdot$ Hélène Jeulin $^{4} \cdot$ François Goehringer $^{5} \cdot$ Gilles Karcher $^{1}$. \\ Laetitia Imbert ${ }^{1,2}$. Pierre-Yves Marie ${ }^{1,6,7}$
}

Received: 9 October 2020 / Accepted: 29 November 2020 / Published online: 8 January 2021

(C) The Author(s), under exclusive licence to Springer-Verlag GmbH, DE part of Springer Nature 2021

\begin{abstract}
Background This study aimed to analyze the rates of tracheobronchitis signs observed on the ventilation scans of COVID-19 patients with shortness of breath, with comparisons to a non-COVID population.

Methods Lung scintigraphy was collected in 10 such COVID patients, as well as from a non-COVID population investigated outside the epidemic wave period, on a CZT-SPECT/CT system, with ventilation images recorded with ${ }^{99 \mathrm{~m}} \mathrm{Tc}$-labeled Technegas ${ }^{\circledR}$ and perfusion images with ${ }^{99 \mathrm{~m}}$ Tc-labeled albumin macroaggregates.

Results A diffuse tracheobronchial uptake was observed on the ventilation scans from 3 COVID patients (30\%), whereas this rate was 3\% (3/90) in the non-COVID group $(P=0.013)$. These 3 patients had no laryngeal extension of Technegas ${ }^{\circledR}$ uptake and limited parenchymal lung abnormalities. Follow-up scintigraphy demonstrated the withdrawal of tracheobronchitis signs in two cases, and the advent of a severe pulmonary embolism in one.

Conclusion Signs of tracheobronchitis may constitute the principal finding on lung SPECT/CT images of COVID-19 patients with shortness of breath.
\end{abstract}

Keywords COVID-19 $\cdot$ Lung scintigraphy $\cdot$ Tracheobronchitis $\cdot$ Dyspnea $\cdot$ CZT-SPECT $\cdot$ CT

Tracheobronchitis is commonly observed at autopsy in COVID-19 patients [1,2], likely in line with a local high expression of angiotensin-converting enzyme 2 receptors (ACE2) [3].

This article is part of the Topical Collection on Infection and inflammation

Pierre-Yves Marie

py.marie@chru-nancy.fr

1 Department of Nuclear Medicine, Nancyclotep, CHRU-Nancy, Université de Lorraine, Nancy, France

2 Université de Lorraine, INSERM, IADI, Nancy, France

3 Department of Radiology, CHRU-Nancy, Université de Lorraine, Nancy, France

4 Department of Virology, CHRU-Nancy, Université de Lorraine, Nancy, France

5 Department of Infectious Diseases, CHRU-Nancy, Nancy, France

6 Université de Lorraine, INSERM, DCAC, Nancy, France

7 Médecine Nucléaire, Hôpital de Brabois, CHRU-Nancy, rue Morvan, 54500 Vandoeuvre-les-Nancy, France
A case report suggested that tracheobronchitis could be detected in the COVID patients through a tracheobronchial uptake of radioactive particles inhaled for lung scintigraphy [4]. These particles tend to deposit and create "hot spots" in the central airways when airway resistance is increased, especially during bronchitis and chronic pulmonary obstructive disease (COPD) [5, 6].

The present study aimed to analyze the rates and associated factors of such signs of tracheobronchitis in the COVID patients referred to ventilation/perfusion lung scintigraphy with shortness of breath, suspected of pulmonary embolism, with comparison to a non-COVID population.

\section{Materials and methods}

We report a series of 10 consecutive patients with COVID-19, experiencing shortness of breath and referred to lung scintigraphy for suspected pulmonary embolism during the period of the COVID-19 outbreak (March 25 to May 15, 2020). One patient has been the subject of a previous case report [4]. The diagnosis of COVID-19 was ascertained by positive reverse 
transcriptase-polymerase chain reaction tests in 7 patients, and by serologic tests in 3 (Lateral Flow Immunoassay, BioSynex®, Illkirch-Graffenstaden, France).

A non-COVID group was constituted of 90 consecutive patients referred to lung scintigraphy on the same CZT camera for a suspected pulmonary embolism, but when viral circulation had become very low (from June 1st to September 1st, 2020). None had any clinical suspicion of COVID-19.

Lung imaging was acquired on a CZT-SPECT/CT system (Veriton ${ }^{\circledR}$, Spectrum Dynamics Medical), starting with CT image acquisition.

Ventilation images were recorded over a 10-min period, after inhalation of ${ }^{99 \mathrm{~m}} \mathrm{Tc}$-labeled Technegas ${ }^{\circledR}$ during two to three consecutive deep inspirations [6], and perfusion scans were acquired over a 3-min period after injection of approximately $730 \mathrm{MBq}$ of ${ }^{99 \mathrm{~m}} \mathrm{Tc}$-labeled albumin macroaggregates [6]. SPECT images were reconstructed with an OSEM iterative method adapted to the collimator system and displayed through $2.46-\mathrm{mm}^{3}$ cubic voxels.

The total procedure time was 30 min overall when including the time required for patient handling and disinfection of the camera and materials.

Quantitative data were represented as mean $\pm \mathrm{SD}$ and compared with ANOVA tests. Qualitative data were expressed as percentages and compared with Fisher exact tests.

\section{Results}

The mean age of the COVID patients was $67 \pm 16$ years, and all but 2 had oxygen supply (Table 1). None had any evident sign of pulmonary embolism on scintigraphy, but a tracheal and/or bronchial uptake was observed on the ventilation scans from 5 patients (Fig. 1, left panel), with uptake being unusually extended on both trachea and bronchi in 3 (numbers 1 to 3 ).

CT was considered subnormal in one of these 3 presumed tracheobronchitis cases (number 1), and the other 2 only exhibited very limited ground-glass opacities (numbers 2 and 3 ). In contrast, all other COVID patients had more extensive CT abnormalities extending to both lungs (Fig. 1).

There was no difference between the 3 COVID patients with, and those without, presumed tracheobronchitis regarding age ( $59 \pm 9$ years vs. $71 \pm 18$ years), the delay from symptom onset ( $16 \pm 12$ days vs. $19 \pm 14$ days), male gender $(67 \%$ vs. $71 \%$ ), active smoking ( $33 \%$ vs. $43 \%$ ), or history of COPD (33\% vs. $14 \%$ ). It is of note, however, that RT-PCR was negative for 2 among these 3 COVID patients (67\%), whereas for only one among the 7 other patients (14\%).

The rate of Technegas ${ }^{\circledR}$ uptake involving both trachea and bronchi was only $3.3 \%$ in the non-COVID group (3/90) and, thus, much lower than in the COVID group $(30 \%, P=0.019)$ (Table 2).

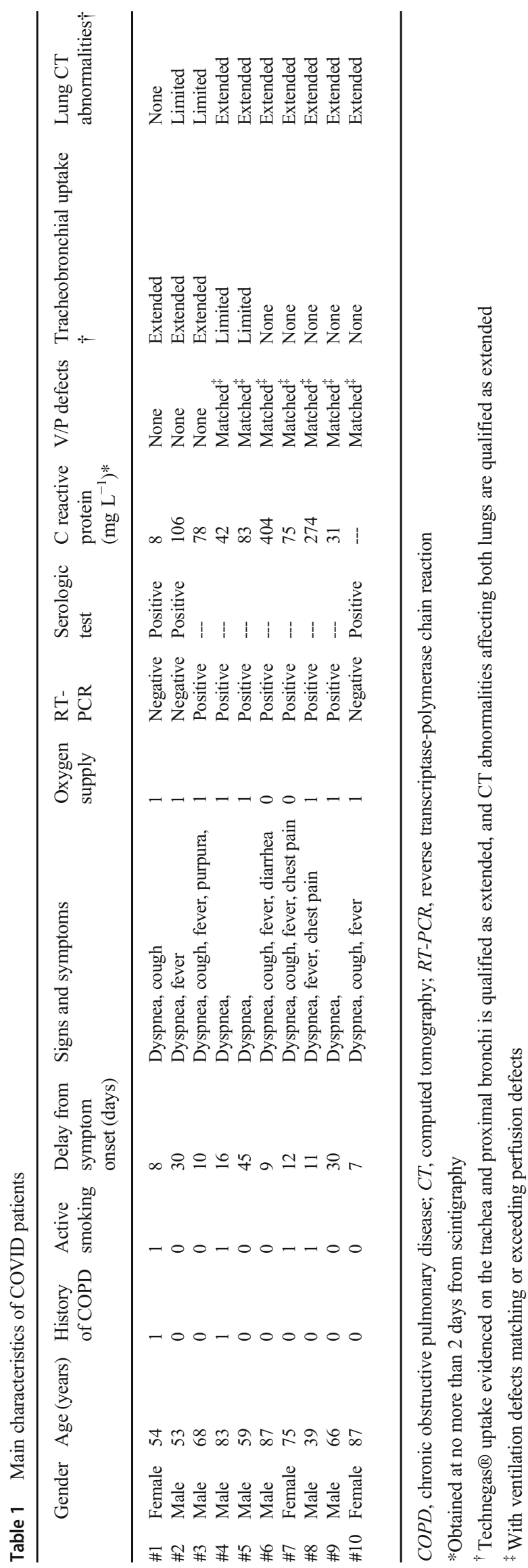




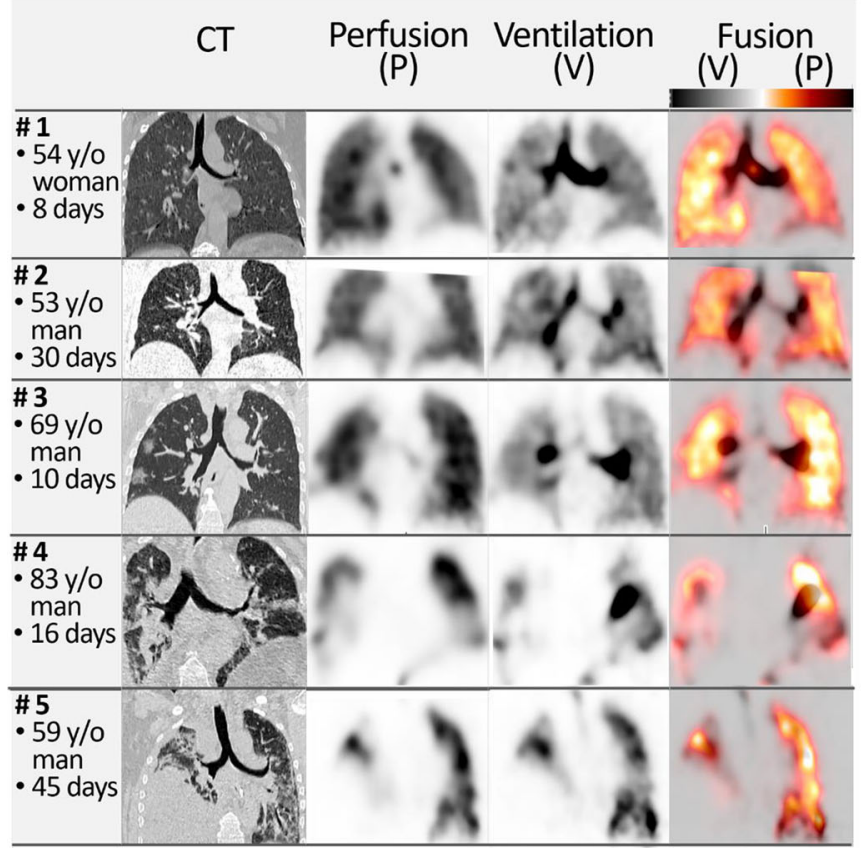

Fig. 1 Illustrative baseline SPECT/CT slices, displayed on a coronal plane passing through the trachea and proximal bronchi, in patients with (left panel) or without (right panel) significant tracheal and/or bronchial Technegas ${ }^{\circledR}$ uptake. The uptake is particularly intense, diffuse, and unusual in patient numbers 1 to 3 , presumably corresponding to a severe tracheobronchitis. Age, gender, and delay

Besides, in the 3 COVID patients with presumed tracheobronchitis, the tracheal uptake had limited upper extension on the larynx and pharynx (Fig. 2). In contrast, such upper extension was documented in all cases of tracheal uptake in the non-COVID group. This suggests a different mechanism of inadequate Technegas ${ }^{\circledR}$ inhalation for these latter.

COVID patient number 1, who had a presumed tracheobronchitis but subnormal CT, had a second SPECT/ CT 1 month later, owing to dyspnea's recurrence 1 week after discontinuation of anticoagulant treatment (Fig. 3). The latter images demonstrated decreased signs of tracheobronchitis

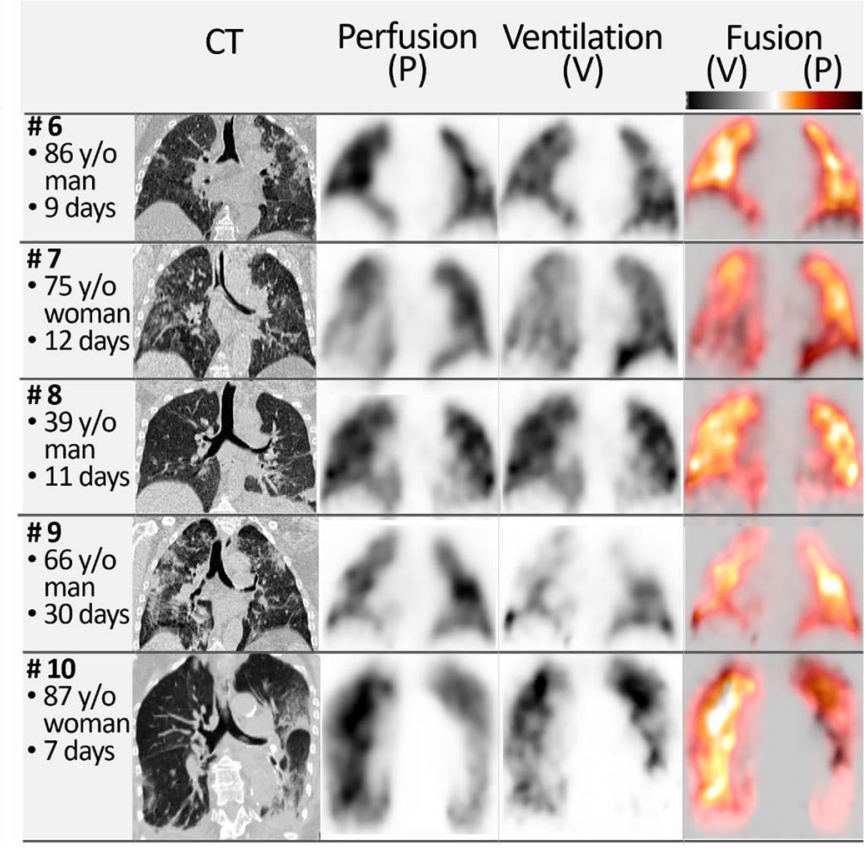

from symptom onset are given in the initial columns. Lung CT images are displayed with a gray scale, whereas perfusion and ventilation images are displayed separately with gray scales and additionally, through a fusion of ventilation images (gray scale) with perfusion images (color scale)

(red arrows), with the advent of pulmonary emboli over the right inferior lobe - i.e., with perfusion defects exceeding ventilation defects and with CT signs of pulmonary infarction and atelectasis (blue arrows). A small mismatch area could be detected at the same site on a reanalysis of the baseline SPECT images from this patient (blue arrow).

A withdrawal of signs of tracheobronchitis or pulmonary embolism was documented on additional follow-up scintigraphies from COVID patient numbers 1 and 2. Finally, at the 34 months follow-up stage, the 3 COVID patients with presumed tracheobronchitis were no longer, or were only poorly, symptomatic.
Table 2 Comparisons of clinical and imaging characteristics between the COVID and nonCOVID groups

\begin{tabular}{llll}
\hline & COVID $(n=10)$ & Non-COVID $(n=90)$ & $P$ value \\
\hline Age (years) & $67 \pm 16$ & $68 \pm 17$ & 0.89 \\
Male gender & $7(70 \%)$ & $35(39 \%)$ & 0.09 \\
Pulmonary embolism at scintigraphy & $0(0 \%)$ & $17(19 \%)$ & 0.20 \\
Abnormal CT scan & $9(90 \%)$ & $59(54 \%)$ & 0.042 \\
Ventilation scans & & & 1.00 \\
$\quad$ Inadequate for diagnostic purposes* & $0(0 \%)$ & $2(2 \%)$ & 0.59 \\
Foci on only trachea & $1(10 \%)$ & $7(8 \%)$ & 1.00 \\
Foci on only bronchi & $1(10 \%)$ & $11(12 \%)$ & 0.013 \\
Foci on both trachea and bronchi & $3(30 \%)$ & $3(3 \%)$ & \\
\hline
\end{tabular}

*Low count images due to an inadequate ventilation, therefore not possible to analyze 
Fig. 2 Anterior maximum intensity projection (MIP) images obtained from the ventilation scans of the 3 COVID patients with uptake on both trachea and bronchi (numbers 1,2, and 3), of the 3 non-COVID patients with uptake on both trachea and bronchi (numbers 11, 12, and 13), and of representative examples of non-COVID patients with uptake on trachea (numbers 21, 22, and 23 ) or bronchi (numbers 31,32 , and 33). A schematic

representation of the

corresponding uptake areas is additionally displayed in the right column. Note the

tracheobronchial uptake of the COVID patients has the particularity of being restricted to proximal bronchi and trachea, without significant extension to the larynx or distal bronchi, thus corresponding to areas with high ACE2 expression [3] and to a presumed tracheobronchitis
Anterior MIP images

Uptake areas

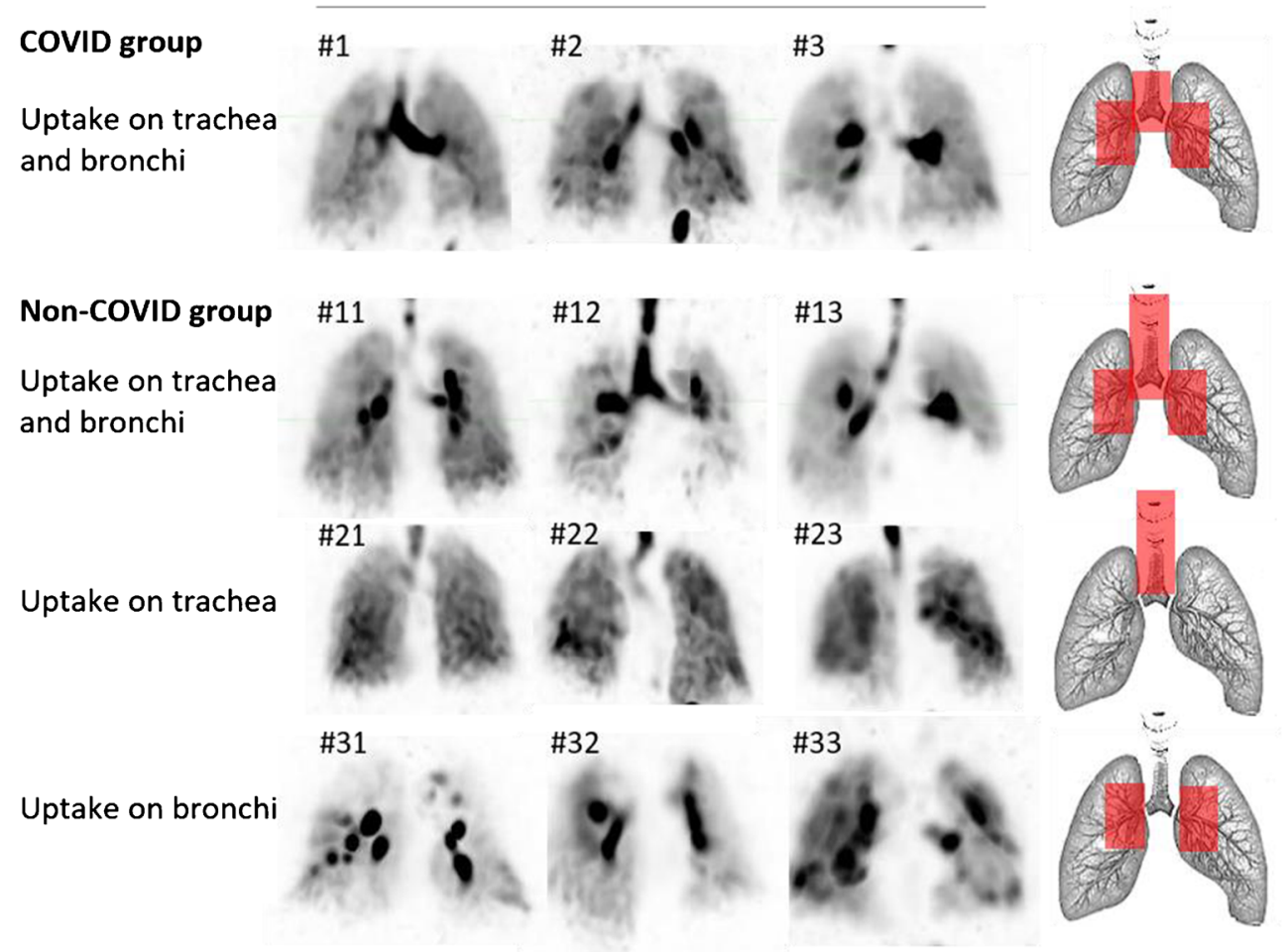

\section{Discussion}

The signs evocative of a COVID-related tracheobronchitis, presented herein and specifically involving areas with high ACE2 expression [3], are very different from bronchial foci commonly observed in COPD patients [5, 6], as well as in our non-COVID population (Fig. 2). The extension of our presumed cases of tracheobronchitis was remarkable here, tending to explain shortness of breath and the need for oxygen supply and contrasting with limited parenchymal lung abnormalities.
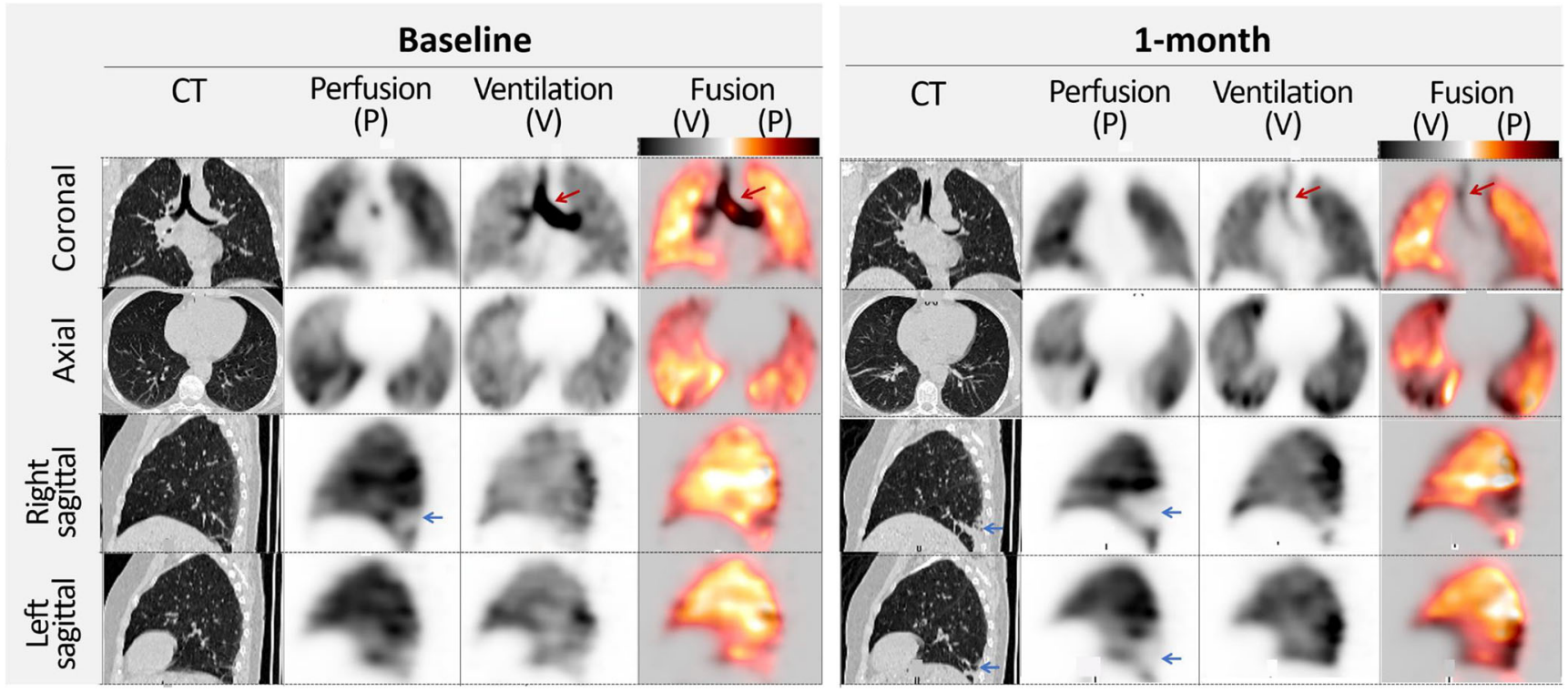

Fig. 3 SPECT/CT images of patient number 1 recorded at baseline (left panel) and 1 month later (right panel), with the representation of different orientations of lung CT slices, as well as the corresponding perfusion and ventilation slices displayed separately with gray scales and additionally,

through a fusion of ventilation images (gray scale) with perfusion images (color scale). See text for additional information. Areas of presumed tracheobronchitis are indicated with red arrows and areas of pulmonary embolism with blue arrows 
These observations compare well with recent results from an international autopsy series of COVID-19 patients [2]. As many as $76 \%$ of these patients had a diffuse tracheobronchitis, and $35 \%$ had both an acute and diffuse form, a proportion that is close to the $30 \%$ observed herein [2]. In addition, tracheobronchitis was commonly associated with signs of a local viral persistence [2].

Such severe and extended forms of tracheobronchitis have already been documented in patients dying from an influenza A/H1N1 viral infection [7] and also in patients with rheumatoid arthritis and ulcerative colitis, where it was found reversible under corticosteroids $[8,9]$.

The reason why COVID-19 predominates on the upper instead of the lower respiratory tract of some of our patients is presently unknown, but this predominance does not imply a low severity, as evidenced by the case with subsequent pulmonary embolism.

COVID-19-related pulmonary emboli are known to predominate on small pulmonary arteries [2]. This is likely why they exhibited a particular evolution on our scintigraphies, with a limited sub-segmental onset and a subsequent extension (Fig. 3).

A limitation is that no direct confirmation of tracheobronchitis was obtained on bronchoscopy, due to the known risk of acute exacerbation of the respiratory condition in patients with severe tracheobronchitis [9]. However, a causal link between the tracheobronchial uptake of Technegas ${ }^{\circledR}$ and diseases of the upper respiratory tract has been extensively established $[5,6]$.

It must also be noted that a few non-COVID patients exhibited a Technegas ${ }^{\circledR}$ uptake involving both trachea and bronchi (3\%), and this was likely explained by a different mechanism of inadequate inhalation of Technegas ${ }^{\circledR}$ (i.e., with the tracheal uptake being extended to the larynx (Fig. 2)).

The incidence of such presumed severe tracheobronchitis was 30\% herein, but the corresponding $95 \%$ confidence interval is very large given the limited sample size, ranging from 11 to $60 \%$. Furthermore, this incidence was observed here in a particular COVID population, mostly including patients with significant symptom aggravation. Thereby, this incidence is presumably different and much lower in more general COVID-19 populations.

Therefore, this observational study suggests that in COVID-19 patients with shortness of breath, a scintigraphy pattern of a presumed tracheobronchitis may constitute the main if not the only sign of respiratory involvement, further identifying a new potential therapeutic target which may require specific treatments.

Acknowledgments The authors wish to thank Ms. Justine McQuillan for the critical review of the manuscript.
Authors' contributions All authors contributed significantly to the analysis and interpretation of the data (AB, HJ, DM, FG, AV, PYM) and/or to the writing of the manuscript (AB, LI, AV, PYM) and/or to the revision of the manuscript (LI, AV, DM, GK, PYM).

Data availability All data are available on request.

\section{Compliance with ethical standards}

Conflict of interest The authors declare that they have no conflict of interest.

Ethics approval and consent to participate All procedures performed in this study were in accordance with the ethical standards of the institutional and/or national research committees and with the 1964 Helsinki declaration and its latest amendments. All patients coming to our department receive a specific document where it is stipulated that their medical data may be used for research purposes, and they may formally object to this on the same document. However, none of the study patients indicated such an objection. In addition, informed consent was retrospectively obtained by telephone calls to all patients for whom SPECT and/or CT images were presented in the articlei.e., all the COVID patients and the non-COVID patients whose SPECT images are displayed in Fig. 2; the only exceptions were 3 patients who died within days following the scintigraphy (1 COVID patient and 2 non-COVID patients). Approval was obtained from our Ethics Committee (Comite éthique du CHRU-Nancy) in light of the retrospective nature of the study and considering that all procedures performed were part of routine care.

Consent for publication Not applicable.

Code availability Not applicable.

\section{References}

1. Calabrese F, Pezzuto F, Fortarezza F, Hofman P, Kern I, Panizo A, et al. Pulmonary pathology and COVID-19: lessons from autopsy. The experience of European Pulmonary Pathologists. Virchows Arch. 2020;477(3):359-72.

2. Borczuk AC, Salvatore SP, Seshan SV, Patel SS, Bussel JB, Mostyka M, et al. COVID-19 pulmonary pathology: a multiinstitutional autopsy cohort from Italy and New York City. Mod Pathol. 2020;2:1-13.

3. Ren X, Glende J, Al-Falah M, de Vries V, de Vries V, SchwegmannWessels C, et al. Analysis of ACE2 in polarized epithelial cells: surface expression and function as receptor for severe acute respiratory syndrome-associated coronavirus. J Gen Virol. 2006;87:16915.

4. Verger A, Bahloul A, Melki S, Karcher G, Imbert L, Marie PY. Tracheobronchitis signs observed on ventilation lung scintigraphy during the course of COVID-19 infection. Eur J Nucl Med Mol Imaging. 2020;47(11):2709-10.

5. Mortensen J, Berg RMG. Lung scintigraphy in COPD. Semin Nucl Med. 2019;49(1):16-21.

6. Bajc M, Schümichen C, Grüning T, Lindqvist A, Le Roux PY, Alatri $\mathrm{A}$, et al. EANM guideline for ventilation/perfusion single-photon emission computed tomography (SPECT) for diagnosis of pulmonary embolism and beyond. Eur J Nucl Med Mol Imaging. 2019;46: 2429-51. 
7. Gill JR, Sheng ZM, Ely SF, Guinee DG, Beasley MB, Suh J, et al. Pulmonary pathologic findings of fatal 2009 pandemic influenza A/ H1N1 viral infections. Arch Pathol Lab Med. 2010;134(2):235-43.

8. Hiyoshi M, Kawai K, Shibuya M, Ozawa T, Kishikawa J, Nirei T, et al. Tracheobronchitis with dyspnea in a patient with ulcerative colitis. Intern Med. 2015;54(7):749-53.
9. Kajikawa S, Noda K, Nozaki Y. Necrotizing tracheobronchitis associated with rheumatoid arthritis. Respir Med Case Rep. 2016;20:313.

Publisher's note Springer Nature remains neutral with regard to jurisdictional claims in published maps and institutional affiliations. 